\title{
Role of T-Type Calcium Current in Identified D-Hair Mechanoreceptor Neurons Studied In Vitro
}

\author{
Anne-Sophie Dubreuil, ${ }^{1}$ Hassan Boukhaddaoui, ${ }^{1}$ Gilles Desmadryl, ${ }^{1}$ Carlos Martinez-Salgado, ${ }^{2}$ Rabih Moshourab, ${ }^{2}$ \\ Gary R. Lewin, ${ }^{2}$ Patrick Carroll, ${ }^{1}$ Jean Valmier, ${ }^{1}$ and Frédérique Scamps ${ }^{1}$ \\ ${ }^{1}$ Institut National de la Santé et de la Recherche Médicale, Unité 583, Hopital St. Éloi, 34091 Montpellier, France, and ${ }^{2}$ Growth Factor and Regeneration \\ Group, Max Delbruck Institute for Molecular Medicine, D-13122 Berlin-Buch, Germany
}

Different subsets of dorsal root ganglion (DRG) mechanoreceptors transduce low- and high-intensity mechanical stimuli. It was shown recently that, in vivo, neurotrophin-4 (NT-4)-dependent D-hair mechanoreceptors specifically express a voltage-activated T-type calcium channel $\left(\mathrm{Ca}_{\mathrm{v}} 3.2\right)$ that may be required for their mechanoreceptive function. Here we show that D-hair mechanoreceptors can be identified in vitro by a rosette-like morphology in the presence of NT-4 and that these rosette neurons are almost all absent in DRG cultures taken from NT-4 knock-out mice. In vitro identification of the D-hair mechanoreceptor allowed us to explore the electrophysiological properties of these cells. We demonstrate that the T-type $\mathrm{Ca}_{\mathrm{v}} 3.2$ channel induced slow membrane depolarization that contributes to lower the voltage threshold for action potential generation and controls spike latency after stimulation of D-hair mechanoreceptors. Indeed, the properties of the T-type amplifier are particularly well suited to explain the high sensitivity of D-hair mechanoreceptors to slowly moving stimuli.

Key words: T-type calcium channel; $\mathrm{Ca}_{\mathrm{v}} 3.2$; neurotrophin; mechanotransduction; D-hair neuron; dorsal root ganglion

\section{Introduction}

The DRG contains a variety of somatic sensory mechanoreceptors that transduce mechanical stimuli. By virtue of their response thresholds, dynamic sensitivities, and adaptation properties, different mechanoreceptors are tuned to detect different aspects of somatic stimuli, such as stimulus velocity, innocuous static indentation, or noxious mechanical stimuli. It has been assumed that the active membrane properties of different receptors determine at least in part their ability to tune into specific stimulus modalities. However, until recently, there has been a conspicuous absence of specific molecular or morphological markers that might allow one to reliably distinguish physiological distinct mechanoreceptor subpopulations in culture in which membrane currents can best be studied.

One subpopulation of highly sensitive, rapidly adapting mechanoreceptors found in all vertebrate species studied to date, including man and mouse, are the so-called D-hair mechanoreceptors (Adriaensen et al., 1983; Koltzenburg et al., 1997). Recently, expression of the T-type calcium channel $\left(\mathrm{Ca}_{\mathrm{v}} 3.2\right)$ has been identified as the first in vivo molecular marker of this sub-

Received April 27, 2004; revised Aug. 16, 2004; accepted Aug. 16, 2004.

We acknowledge support from the Association Française contre les Myopathies, the Deutsche Forschungsgemeinschaft, a European Union Marie Curie fellowship to C.M.-S., and a Deutscher Akademischer Austausch Dienst stipend to R.M. We thank Jing Hu for help in establishing D-hair stimulation protocols.

Correspondence should be addressed to Frédérique Scamps, Institut National de la Santé et de la Recherche Médicale, Unité 583, Institut des Neurosciences de Montpellier, 80 Avenue Augustin Fliche, Boîte Postale 74103, 34091 Montpellier Cedex 5, France. E-mail: scamps@univ-montp2.fr.

C. Martinez-Salgado's present address: Unidad de Investigación, Hospital Universitario de Salamanca, 37007 Salamanca, Spain.

DOI:10.1523/JNEUROSCI.1598-04.2004

Copyright $\odot 2004$ Society for Neuroscience $\quad$ 0270-6474/04/248480-05\$15.00/0 population (Shin et al., 2003). D-hair mechanoreceptors also express high levels of the TrkB receptor and die in the absence of the TrkB ligand neurotrophin-4 (NT-4) (Stucky et al., 2002). T-type calcium channels represent a class of voltage-activated calcium channel present in most excitable cells, including embryonic and adult sensory neurons (Bossu et al., 1985; Desmadryl et al., 1998), and are encoded by three T-type $\alpha 1$ subunit genes, $\alpha 1 \mathrm{G}\left(\mathrm{Ca}_{\mathrm{v}} 3.1\right)$, $\alpha 1 \mathrm{H}\left(\mathrm{Ca}_{\mathrm{v}} 3.2\right)$, and $\alpha 1 \mathrm{I}\left(\mathrm{Ca}_{\mathrm{v}} 3.3\right)$ (Perez-Reyes, 1999). Pharmacological experiments using the T-type channel antagonist mibefradil on identified D-hair receptors recorded in the skin indicated that the $\mathrm{Ca}_{\mathrm{v}} 3.2$ channel might be required for the normal function of the D-hair mechanoreceptor (Shin et al., 2003). One drawback of the skin nerve preparation is that voltage-gated membrane currents cannot be studied directly with intracellular recording methods.

Here we show that D-hair mechanoreceptors can be simply identified by their rosette-like morphology in cultures of adult DRG neurons in the presence of NT-4. All rosette neurons express giant T-type calcium current and are almost all absent in cultures taken from adult NT-4 mutant mice. Identification of the D-hair mechanoreceptor in vitro has allowed us to show how T-type channels can support the high mechanical sensitivity of these cells. Specifically, we present evidence that the T-type conductance may amplify receptor potentials especially to slowly moving stimuli to which D-hair receptors are particularly well tuned (Brown and Iggo, 1967).

\section{Materials and Methods}

Culture and morphological analysis. Neuronal cultures were established from lumbar dorsal root ganglia of 5- to 10-week-old female Swiss mice or knock-out mice homozygous for deletion of the NT-4 gene as reported 
previously (Andre et al., 2003). NT-4 mutant mice were originally obtained from The Jackson Laboratory (Bar Harbor, ME). Two hours after plating, the culture medium was carefully removed and replaced to eliminate dead cells and tissue debris, and recombinant NT-4 was added (PeproTech, London, UK).

For morphometric analysis, cells were fixed and stained with the rabbit polyclonal anti-neurofilament 200 (1:400; Sigma, Saint Quentin, Fallavier, France) tagged with an FITC-conjugated goat anti-rabbit antibody [1:200; Jackson ImmunoResearch (West Grove, PA) and Interchim (Montlucon, France)]. Image acquisition was performed with a CCD camera (Rover) and analyzed using MetaMorph software (Universal Imaging Corporation, West Chester, PA).

Electrophysiology. Voltage-gated calcium currents $\left(I_{\mathrm{Ca}}\right)$ and action potentials in dorsal root ganglion neurons were recorded after $1 \mathrm{~d}$ in vitro, at $20-22^{\circ} \mathrm{C}$. Whole-cell patch-clamp recordings were made under conditions optimized for the isolation of $I_{\mathrm{Ca}}$ from other voltage-activated currents (Andre et al., 2003). For action potential recordings, the bathing solution contained $140 \mathrm{~mm} \mathrm{NaCl}$ and $5 \mathrm{~mm} \mathrm{KCl}$ in place of tetraethylammonium chloride, and, for intracellular solution, $\mathrm{CsCl}$ was replaced with $145 \mathrm{~mm} \mathrm{KCl}$. Signals were filtered at $5 \mathrm{kHz}$ and sampled at $10 \mathrm{kHz}$. For each experiment, cell size was estimated by means of an eyepiece micrometer scale. Data are expressed as means \pm SEM. Statistical evaluations are based on ANOVA, paired or unpaired $t$ test as appropriate. A value of $p<0.05$ was considered statistically significant.

In vitro single fiber recording. An in vitro skin-nerve preparation was used to record from functionally single primary afferents in microdissected teased filaments of the saphenous nerve as described previously (Koltzenburg et al., 1997). D-hair mechanoreceptors were identified by their distinctive high mechanosensitivity and slow conduction velocity $(<10 \mathrm{~m} / \mathrm{sec})$.

\section{Results}

\section{NT-4 induced a typical rosette growth in D-hair neurons in vitro}

Identification of D-hair neurons in vitro was based on the fact that these neurons express TrkB, the high-affinity BDNF and NT-4 tyrosine kinase receptor, and selectively die in NT-4deficient mice in adulthood (Stucky et al., 2002). In the first step, we used the capacity of neurotrophins to induce different growth types in adult DRG neurons in vitro to morphologically identify NT-4-sensitive neurons (Lindsay, 1988). After 24 hr in vitro, neurons from NT-4 (10 ng/ml)-treated wells were fixed and stained with anti-neurofilament and compared using morphometric analysis. Morphological comparison between control and NT-4treated neurons allowed us to observe that the addition of NT-4 induced a particular mode of growth in a subpopulation of medium-sized neurons that represented $4.8 \pm 0.5 \%$ of the total number of DRG neurons (35 of 691 from three cultures). As shown in Figure $1 A$, these neurons displayed a typical rosette of highly branched and varicose neurites. On 11 well isolated neurons, the mean diameter of the soma was $38 \pm 3 \mu \mathrm{m}$, with a mean radius (defined as the distance from the center of the cell soma to end of the farthest neurite) of $138 \pm 13 \mu \mathrm{m}$. The number of branching points per neuron was $62 \pm 16$. The ratio of the number of branching points versus radius was dramatically increased in rosette neurons compared with other neurons in the same cell soma diameter range, $0.5 \pm 0.1(n=11)$ and $0.05 \pm 0.02(n=9)$, respectively $(p<0.01)$. Such growth could also be induced by addition of $10 \mathrm{ng} / \mathrm{ml}$ BDNF $(3.8 \pm 1.0 \%$ of total number of neurons; $n=2$ cultures) but was not reliably observed in control neurotrophin-free conditions or in the presence of NT-3 or NGF (both $10 \mathrm{ng} / \mathrm{ml}$ ) (data not shown). Because it has been reported that, in adulthood, NT-4 ${ }^{-1-}$ mice specifically lose the D-hair mechanoreceptors, we established DRG cultures from 10-weekold NT-4 ${ }^{-1-}$ mice. Morphological analysis shows that the main difference from wild-type mice was a significant decrease of ro-
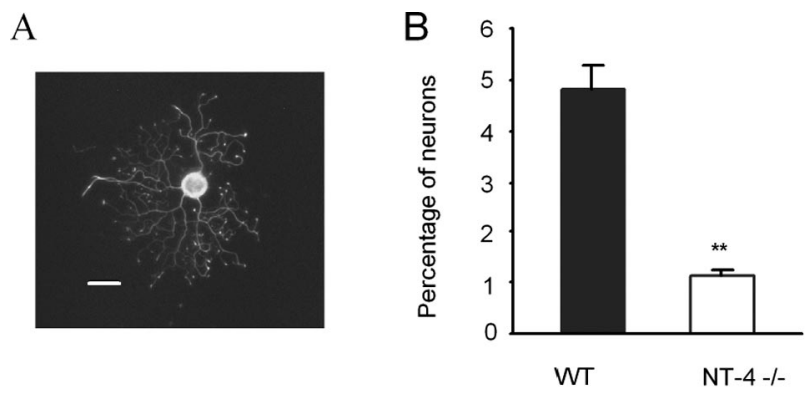

C

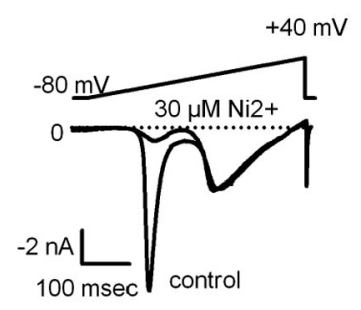

D

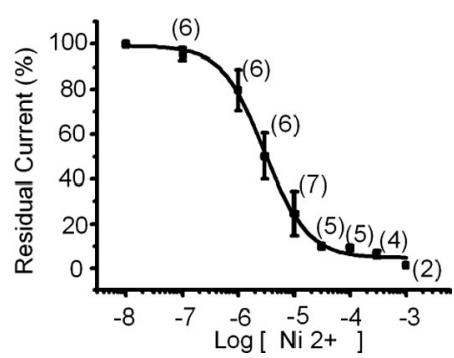

Figure 1. Morphological and functional identification of D-hair neurons in vitro. A, Neurofilament staining of a neuron with a rosette-like morphology induced by incubation with 10 $\mathrm{ng} / \mathrm{mI}$ NT-4. Scale bar, $40 \mu \mathrm{m}$. B, The percentage of rosette neurons was calculated relative to the total neuronal population and was significantly reduced in NT-4 $4^{-1-}$ mutant mice. WT, Wild type. C, A ramp protocol applied to a rosette neuron from -80 to $+40 \mathrm{mV}, 500 \mathrm{msec}$ duration with the whole-cell patch-clamp technique showed the presence of a high-amplitude low-voltage-activated $I_{\mathrm{Ca}}$ compared with high-voltage-activated $I_{\mathrm{Ca}}$. This calcium current profile was observed exclusively in the rosette-like neurons. D, Dose-response curve of $\mathrm{NiCl}_{2}$ inhibition of low-voltage-activated $I_{\mathrm{Ca}}$. The inhibitory effect of Ni was evaluated on calcium currents elicited with ramp protocols. Dose-response curve was fitted with a Hill equation: $y=V_{\max } \times$ $\left\{\mathrm{Ni}^{n} /\left(K_{1 / 2}+\{\mathrm{Ni}\}\right)^{n}\right.$ (dashed line). From fit of the data, the $\mathrm{Ni}^{2+}$ concentration for halfmaximal effect $K_{1 / 2}$ was $2.9 \pm 0.2 \mu \mathrm{m}$.

sette neurons, from 5 to $1.1 \pm 0.1 \%$ ( 6 of $545 ; n=3$ cultures; $p<$ $0.01)$ after 24 or $48 \mathrm{hr}$ culture incubation with $10 \mathrm{ng} / \mathrm{ml} \mathrm{NT}-4$ (Fig. $1 \mathrm{~B}$ ). In 6 -week-old NT-4 ${ }^{-1-}$ mice, neurons bearing rosette growth were still observable after NT-4 treatment in similar proportions as in wild-type neurons $(3 \%, 6$ of 200 ; from one culture). The low percentage of neurons bearing rosette growth in wild-type mice and their drastic reduction in NT- $4^{-1-}$ mice indicates that these neurons probably represent the D-hair mechanoreceptor cells that selectively die in adult $\mathrm{NT}-4^{-/-}$mice (Stucky et al., 2002; Shin et al., 2003).

Because D-hair cells express high levels of the T-type $\mathrm{Ca}_{\mathrm{v}} 3.2$ calcium channels in vivo, calcium current recordings were performed in neurons displaying the rosette growth induced by a 24 hr treatment with $10 \mathrm{ng} / \mathrm{ml} \mathrm{NT-4}$. Ramp protocols were applied to rapidly screen the low- and high-voltage calcium current profile of these neurons (Fig. 1C). All rosette neurons (mean somatic diameter, $35.5 \pm 3.8 \mu \mathrm{m} ; n=29$ ) displayed a predominant T-type current relative to high-voltage-activated (HVA) $I_{\mathrm{Ca}}$ (mean amplitude, $-5.8 \pm 1.8$ and $-1.2 \pm 0.4 \mathrm{nA}$, respectively; $n=29$ ). Neurons displaying no rosette growth under neurotrophin treatment never expressed predominant T-type calcium current relative to HVA $I_{\mathrm{Ca}}$ (mean amplitude, $-0.3 \pm 0.2$ and $-4.2 \pm 1.2 \mathrm{nA}$, respectively; $n=10$ ). A unique feature of the T-type $\mathrm{Ca}_{\mathrm{v}} 3.2$ calcium channel is a high sensitivity to nickel inhibition (Lee et al., 1999). We thus performed the dose-response curve of $\mathrm{NiCl}_{2}$ inhibition on the low-voltage-activated $I_{\mathrm{Ca}}$ expressed in rosette neurons. As shown in Figure 1, $C$ and $D, 30 \mu \mathrm{M}$ $\mathrm{NiCl}_{2}$ selectively inhibited most T-type $I_{\mathrm{Ca}}$ current without sig- 


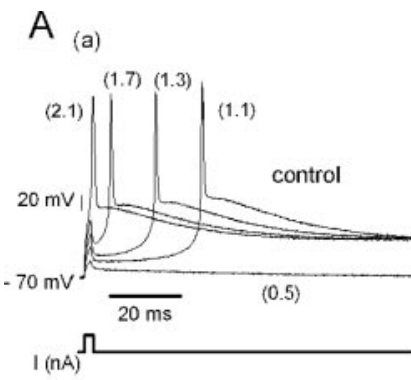

(b)

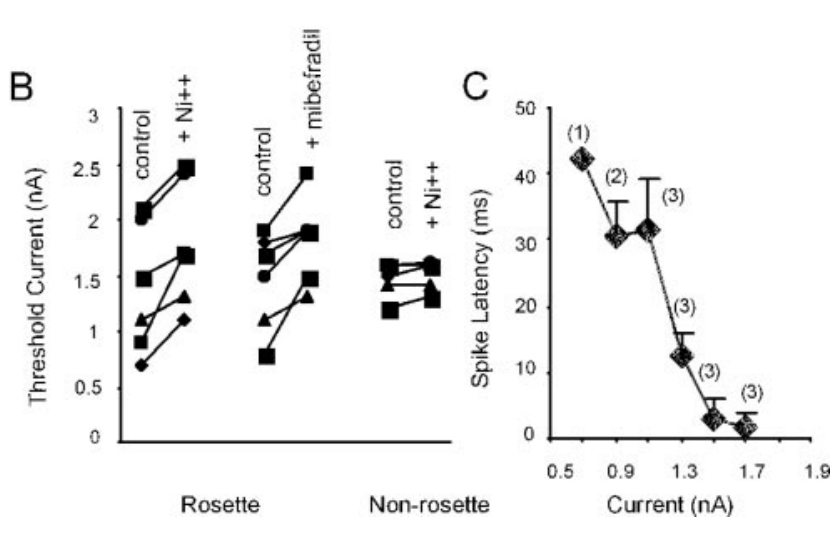

Figure 2. T-type $I_{\mathrm{Ca}}$ increases $D$-hair mechanoreceptor excitability. A, Depolarizing currents at $2 \mathrm{msec}(I)$ of increasing amplitudes were applied to a rosette neuron to trigger an action potential (current amplitudes are given in brackets on voltage traces) under control conditions and after application of $\mathrm{NiCl}_{2}(b)$. Action potential of rosette neurons is characterized by a pronounced afterdepolarization ( $a$ ) inhibited by the application of $30 \mu \mathrm{M} \mathrm{NiCl}(b)$. $B$, Threshold current for an action potential generation in individual rosette neurons is significantly increased after inhibition of T-type $I_{\mathrm{Ca}}$ with $30 \mu \mathrm{M} \mathrm{NiCl}{ }_{2}(n=6$; paired $t$ test; $p=0.01$ ) or $10 \mu \mathrm{m}$ mibefradil ( $n=6$; paired $t$ test; $p=0.02$ ). In non-rosette neurons, threshold current was not changed after application of $30 \mu \mathrm{m} \mathrm{NiCl}$ ( $n=6$; paired $t$ test; $p=0.1$ ). C, In rosette neurons, the latency for action potential generation was measured as the time between peak voltage induced by current injection and fast spike potential. Under control conditions, spike latency was dependent on current amplitude, with longer latency observed with smallest current amplitudes. In $30 \mu \mathrm{m} \mathrm{NiCl}$-treated rosette neurons, latency for spike generation was short $(<1$ msec) and unrelated to current amplitude (see voltage traces in $A$ ).

nificant effects on HVA calcium current. The dose-response curve obtained from ramp protocols was well fitted with a Hill equation, giving a half-inhibitory concentration $\left(\mathrm{IC}_{50}\right)$ of $3 \mu \mathrm{M}$, a value in good agreement with the known pharmacology of recombinantly expressed $\mathrm{Ca}_{\mathrm{v}} 3.2$ channels (Fig. 1D) (Lee et al., 1999). Together, these results identify the rosette neurons in the presence of NT-4 as isolated D-hair mechanoreceptor neurons.

\section{T-type calcium current-induced slow membrane} depolarization accounts for sensitivity and latency of D-hair mechanotransduction

From previous skin-nerve preparation experiments, it is unclear how exactly T-type $I_{\mathrm{Ca}}$ expressed in D-hair mechanoreceptors contributes to the generation of action potentials and how T-type $I_{\mathrm{Ca}}$ underlies the high mechanosensitivity of these cells. To evaluate the role of T-type channel on excitability, we performed current-clamp recordings on cultured rosette neurons in the presence of NT-4. Under our ionic conditions, the resting membrane potential of rosette neurons was $-69 \pm 3 \mathrm{mV}(n=10)$. In an attempt to mimic receptor potential stimulation, brief depolarizing currents of increasing amplitude were applied to elicit fast action potentials under control conditions and after application of $30 \mu \mathrm{M} \mathrm{NiCl}_{2}$ (Fig. $2 \mathrm{~A}$ ). The same concentration of $\mathrm{NiCl}_{2}$ was without effect on voltage-gated sodium currents (data not
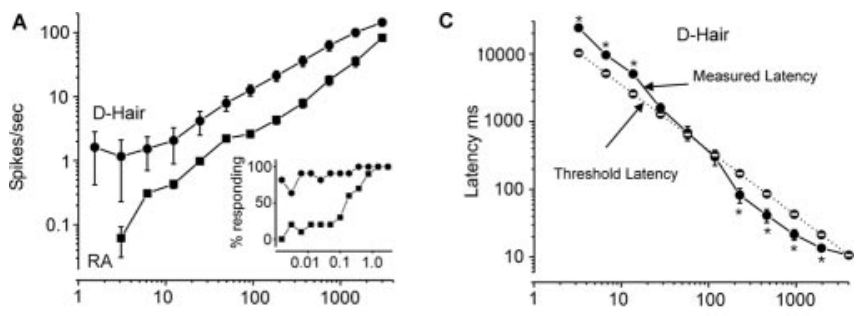

B

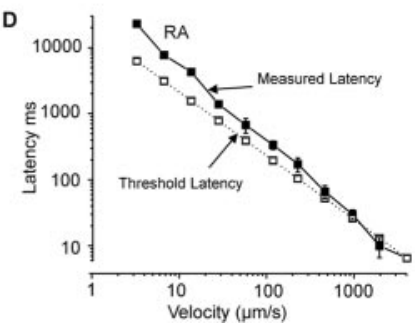

Figure 3. Velocity sensitivity of RA and D-hair mechanoreceptors. Recordings from 11 D-hair and 10 RA mechanoreceptors ( $n=4$ mice) indicate that $D$-hair mechanoreceptors are exquisitely sensitive to movement, in particular, very slow movements (1.5-100 $\mu \mathrm{m} / \mathrm{sec})$. In $A$, the mean firing rate and proportion of neurons responding (inset) to a series of $100 \mu \mathrm{m}$ ramps ranging from 1.5 to $3000 \mu \mathrm{m} / \mathrm{sec}$ is shown for D-hair (black filled circles) and RA (black filled squares) mechanoreceptors. The latency for the first mechanically evoked spike plotted against velocity is plotted separately for $D$-hair ( $C$ ) and RA mechanoreceptors $(D)$. In $B$, a schematic diagram is shown indicating how a theoretical threshold latency was calculated for each neuron. The fastest latency, which was obtained using the fastest movement, was used to extrapolate a threshold value with slower-moving stimuli with the assumption that the indentation needed to evoke the first spike is constant at different ramp on velocities. The threshold latency (open circles or squares) is therefore plotted together with the measured latency to determine whether the first spike is evoked faster or slower than the theoretical value $(C, D)$.

shown). Under control conditions, the action potential of rosette neurons was characterized by a spike, followed by a pronounced afterdepolarization (ADP). $\mathrm{NiCl}_{2}$ at $30 \mu \mathrm{M}$ or $10 \mu \mathrm{M}$ mibefradil application inhibited the ADP and increased the value of threshold current from $1.3 \pm 0.4$ to $1.8 \pm 0.4 \mathrm{nA}$ and from $1.5 \pm 0.3$ to $1.8 \pm 0.2 \mathrm{nA}$, respectively $(n=6 ; p<0.01)$ (Fig. $2 A, B)$. Action potential recordings from medium-sized neurons without rosette growth did not display an ADP, and no change in threshold current was observed after $\mathrm{NiCl}_{2}$ application $(1.5 \pm 0.2$ and $1.6 \pm$ $0.1 \mathrm{nA}$, respectively; $n=6$; NS) (Fig. 2 B). As clearly shown Figure $2 C$, there was a strong correlation between current amplitude injection and latency for action potential initiation in rosette neurons, with a longer latency for the smallest injected current.

The finding that small current injections led to longer latency spikes with a lower threshold for activation than during blockade of T-type currents led us to ask whether a similar behavior could be observed at the receptor endings of D-hair mechanoreceptors in vivo. In the present experiments, we compared the velocity sensitivity of rapidly adapting (RA) mechanoreceptors, which presumably do not express large amplitude T-type current, with that of D-hair mechanoreceptors. The neurons were therefore tested with a series of constant amplitude displacement stimuli $(50,100$, or $150 \mu \mathrm{m})$ with varying ramp velocities of between 1.5 and $3000 \mu \mathrm{m} / \mathrm{sec}$. Remarkably, almost all of the D-hair mechanoreceptors reliably responded to movements as slow as $1.5 \mu \mathrm{m} / \mathrm{sec}$, with mean rates of firing above $1 \mathrm{~Hz}$ (Fig. $3 A$ and inset). RA mechanoreceptors, on the other hand, only reliably code movements that are 100-fold faster $(>100 \mu \mathrm{m} / \mathrm{sec})$, and most of the tested RA neurons $(80 \%)$ do not even respond to movements below $100 \mu \mathrm{m} / \mathrm{sec}$ (Fig. $3 A$ and inset). At any given ramp velocity, the mechanical latency reflects the indentation needed to elicit threshold response. We measured the mechanical latencies at the 


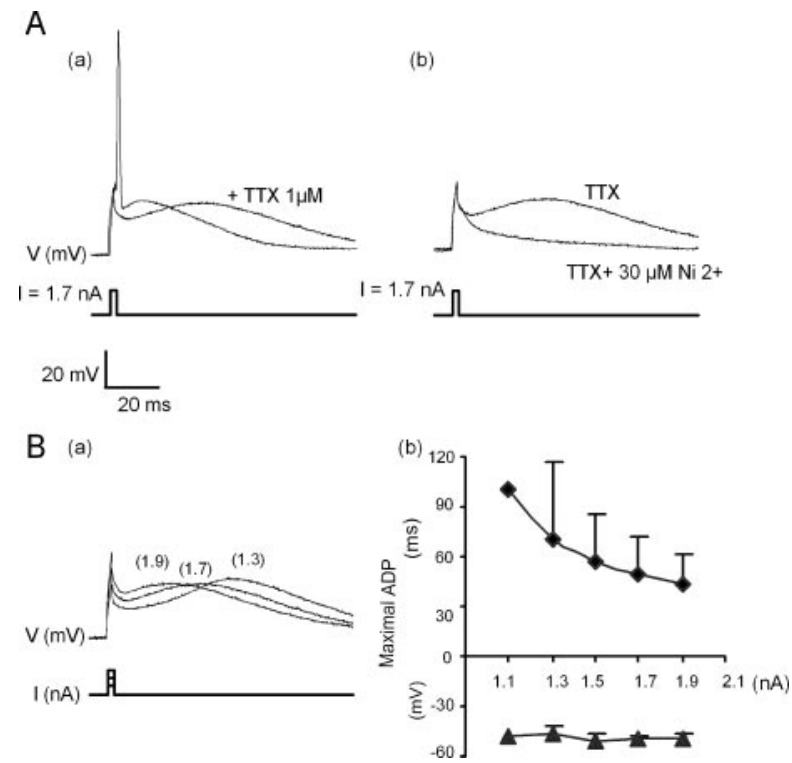

Figure 4. Slow action potential induced by T-type $I_{\mathrm{Ca}}$ accounts for latency in D-hair neurons. A, Application of $1 \mu \mathrm{m}$ TTX inhibited the fast spike of D-hair cell action potential ( $a$ ); addition of $30 \mu \mathrm{m} \mathrm{NiCl}$ in the presence of TTX inhibited the slow depolarization (b). $B$, Rise time, but not maximal amplitude, of slow depolarization is voltage dependent, decreasing with increasing depolarizing current amplitude.

different tested velocities for RA and D-hair mechanoreceptors as a possible indicator of spike latency with weak and strong receptor activation (slow and fast movement). The measured latency was then compared with threshold latency; the latter is defined as the mean latency extrapolated from the shortest latency measured at the fastest velocity for each neuron at each velocity (Fig. $3 B$ ). Interestingly, D-hair mechanoreceptor had much longer mechanical latencies than expected when responding to very slowly moving stimuli (3-10 $\mu \mathrm{m} / \mathrm{sec}$ ) (Fig. 3C) and reacted much faster than predicted from the threshold latency in the range of 150 and $1500 \mu \mathrm{m} / \mathrm{sec}$. RA mechanoreceptors, in contrast, never displayed latencies faster than the threshold latency value at any velocity tested (Fig. 3D). It appeared that RA mechanoreceptors also had longer mechanical latencies to slowly moving stimuli, but most neurons tested did not respond at all to the slow movement (Fig. 3A, inset); the longer measured latencies might reflect an increased displacement threshold for the few responding RA neurons (two of 10 tested) at these slow velocities. In summary, this data indicates that D-hair, but not RA, mechanoreceptors respond reliably but with long latency to moving stimuli that are near threshold. This behavior is strongly reminiscent of the long latency spikes evoked by small current injections into rosette neurons in culture.

To better understand the effect of T-type on action potential generation, $1 \mu \mathrm{M}$ TTX was used to inhibit the fast depolarizing spike of the action potential. Under this condition, no action potentials were evoked but the ADP remained, demonstrating that it was not a consequence of the fast action potential but rather appeared as a slow membrane depolarization. This slow depolarization was inhibited by low $\mathrm{NiCl}_{2}$ concentration, again indicating that its presence required activation of T-type calcium channels. Maximal values of the slow depolarization before and after TTX application were not significantly changed and close to $-45 \mathrm{mV}$; however, the time necessary to reach peak value was significantly longer after sodium-spike inhibition (Fig. 4). Thus, ADP in rosette neurons had the characteristics of a regenerative process, the kinetics of which were voltage dependent.

\section{Discussion}

Here we have identified D-hair mechanoreceptors in culture based on their specific rosette-like growth pattern in the presence of NT-4 and their selective death in NT-4 ${ }^{-1-}$ mice. This is the first demonstration of a simple method to identify a physiologically defined mechanoreceptor population in vitro. We show that, in vitro, these neurons retain at least two prominent in vivo features of D-hair mechanoreceptors. First, they express TrkB, the high-affinity receptor for NT-4 and BDNF (data not shown), and display a functional response to NT-4 and BDNF (Stucky et al., 2002; Shin et al., 2003). Second, they exhibit a prominent T-type $I_{\mathrm{Ca}}$ predicted from the specific expression of $\mathrm{Ca}_{\mathrm{v}} 3.2$ in D-hair mechanoreceptors in vivo (Shin et al., 2003). An open question from previous work was how might T-type calcium channels contribute to the transformation of mechanosensory information into action potential trains in D-hair mechanoreceptors. Here we show, using patch-clamp techniques, that the T-type $I_{\mathrm{Ca}}$ acts as an amplifier of depolarizing events, leading to a decrease in the current threshold for action potential generation in rosette neurons. This amplification accounts for the high sensitivity of D-hair mechanoreceptor neurons, and we present evidence that the T-type $I_{\mathrm{Ca}}$ may especially enhance the remarkable sensitivity of these neurons to very slowly moving stimuli.

The small percentage of rosette neurons in our cultures (5\%), which all have large T-type $I_{\mathrm{Ca}}$ in the presence of NT-4 or BDNF, is remarkably consistent with the percentage of neurons in vivo $(6 \%)$ that were found previously to express large amounts of $\mathrm{Ca}_{\mathrm{v}} 3.2$ and TrkB mRNA (Stucky et al., 2002; Shin et al., 2003). Indeed, just like in vivo, the rosette neurons disappear from cultures taken from older NT-4 knock-out mice that lose D-hair mechanoreceptors through apoptosis with a parallel loss of TrkB and $\mathrm{Ca}_{\mathrm{v}}$ 3.2-positive cells in the ganglion (Stucky et al., 2002; Shin et al., 2003). NT-4 or BDNF treatment did not appear to rescue or induce the differentiation of cells with characteristics of D-hair mechanoreceptors as in control cultures lacking neurotrophins; the same proportion of neurons expressing a large T-type current was found among medium-sized cells (Andre et al., 2003). However, both NT-4 and BDNF induced specific rosette-like growth only in neurons dependent on NT-4 in the mature mouse. It has long been noted that, in contrast to $A \beta$-fiber rapidly adapting mechanoreceptors, D-hair mechanoreceptors respond to the movement of essentially all hairs within their relatively large receptive fields (Brown and Iggo, 1967; Lewin et al., 1992). It may the case that the growth in culture of these neurons recapitulates aspects of their apparently exuberant terminal growth in the skin. This raises the interesting possibility that the peripheral branching pattern of D-hair mechanoreceptors is controlled by neurotrophins during development. However, up to date, there is no definitive published data that identify the morphology of D-hair mechanoreceptor sensory endings in the skin.

Previous patch-clamp studies have demonstrated that a subpopulation of medium-sized DRG neurons displays unusually large T-type currents (Schroeder et al., 1990; Scroggs and Fox, 1992; Andre et al., 2003). Here we demonstrate that only rosette neurons expressed this current in NT-4-treated cultures, and pharmacological characterization of this T-type current suggests that it is related to the $\mathrm{Ca}_{\mathrm{v}} 3.2$ gene product. Indeed, the $\mathrm{IC}_{50}$ for T-type $I_{\mathrm{Ca}}$ inhibition by $\mathrm{NiCl}_{2}$ was $3 \mu \mathrm{M}$ in our experiments and 6 $\mu \mathrm{M}$ for $\alpha 1 \mathrm{H}\left(\mathrm{Ca}_{\mathrm{v}} 3.2\right)$, whereas $250 \mu \mathrm{M} \mathrm{NiCl}_{2}$ is needed for halfinhibition of $\alpha 1 \mathrm{G}\left(\mathrm{Ca}_{\mathrm{v}} 3.1\right)$ and of $\alpha 1 \mathrm{I}\left(\mathrm{Ca}_{\mathrm{v}} 3.3\right)$ (Lee et al., 1999). It is still possible that other T-type calcium channel genes, for example $\mathrm{Ca}_{\mathrm{v}} 3.3$, are coexpressed at low levels in D-hair mechano- 
receptor neurons (Shin et al., 2003). The present data show that medium-sized neurons with giant T-type current are D-hair receptors and that this large T-type current is probably carried by the $\mathrm{Ca}_{\mathrm{v}} 3.2$ subunit gene product.

Low $\mathrm{NiCl}_{2}$ concentrations or mibefradil, a less selective T-type $I_{\mathrm{Ca}}$ blocker, increased the amplitude of the depolarizing current necessary to trigger the sodium action potential without affecting the amplitude of the fast sodium-driven action potentials. These data support the idea that T-type $I_{\mathrm{Ca}}$ carries a sufficient amount of charge to initiate an action potential and controls the voltage threshold that allows spike generation in D-hair mechanoreceptors. Altogether, the present results suggest that, in D-hair mechanoreceptor cells, T-type $I_{\mathrm{Ca}}$ has a role in enhancing the sensitivity to mechanical stimulation by lowering the voltage threshold for action potential generation.

In this study, we have identified a slow depolarizing potential that appears to be dependent on a T-type $I_{\mathrm{Ca}}$, and this slow depolarizing potential most likely contributes to the delayed spike observed with a threshold current injection in rosette neurons. The presence of the slow depolarizing potential in rosette neurons may allow spike generation with very small current injections at the expense of a delayed spike initiation. Using a series of velocity stimuli, which is the adequate stimulus for D-hair mechanoreceptors, we observed that slow moving stimuli that presumably initiate the smallest receptor potentials produce spikes, albeit with a much increased delay compared with faster moving stimuli. Thus, we conclude that the presence of the T-type $I_{\mathrm{Ca}}$ may be especially important in allowing $\mathrm{D}$-hair mechanoreceptors to respond to slow moving stimuli. In vivo action potential recordings from DRG neuron somata did not reveal a T-typedependent ADP (Ritter and Mendell, 1992; Djouhri et al., 1998). Experiments with the skin-nerve preparation indicated that T-type $I_{\mathrm{Ca}}$ was expressed in the receptor terminal but not along the axon (Shin et al., 2003). Although our in vitro data may seem at variance with the these results, several studies have demonstrated a redistribution of channels and receptors in cultured neurons that would normally have been transported to the receptor terminal (Baccaglini and Hogan, 1983; Cesare and Mcnaughton, 1997). Besides its effect on excitability, giant T-type currents probably account for a significant entry of calcium ions, which has the potential to control other ionic conductances. In addition, it is plausible that T-type current-regulated $\mathrm{Ca}^{2+}$ entry will activate intracellular signaling pathways important in the regulation of mechanotransduction. The in vitro model described here will allow the detailed exploration of these questions.

We have demonstrated for the first time that a functional DRG neuron subpopulation of D-hair mechanoreceptors can be simply identified by their particular "rosette" morphology in vitro. This has allowed us to show how T-type channels regulate the high mechanical sensitivity of these cells. Our results establish the T-type $\mathrm{Ca}_{\mathrm{v}} 3.2$ channel as a candidate molecule for the normal functioning of D-hair mechanoreceptor DRG neurons and demonstrate that $\mathrm{Ca}_{\mathrm{v}} 3.2$ acts as an amplifier of electrical stimuli in these cells. This in vitro system opens new perspectives for the study of mechanosensation in D-hair neurons.

\section{References}

Adriaensen H, Gybels J, Handwerker HO, Van Hees J (1983) Response properties of thin myelinated (A-delta) fibers in human skin nerves. J Neurophysiol 49:111-122.

Andre S, Boukhaddaoui H, Campo B, Al-Jumaily M, Mayeux V, Greuet D, Valmier J, Scamps F (2003) Axotomy-induced expression of calciumactivated chloride current in subpopulations of mouse dorsal root ganglion neurons. J Neurophysiol 90:3764-3773.

Baccaglini PI, Hogan PG (1983) Some rat sensory neurons in culture express characteristics of differentiated pain sensory cells. Proc Natl Acad Sci USA 80:594-598.

Bossu JL, Feltz A, Thomann JM (1985) Depolarization elicits two distinct calcium currents in vertebrate sensory neurones. Pflügers Arch 403:360-368.

Brown AG, Iggo A (1967) A quantitative study of cutaneous receptors and afferent fibres in the cat and rabbit. J Physiol (Lond) 193:707-733.

Cesare P, Mcnaughton P (1997) Peripheral pain mechanisms. Curr Opin Neurobiol 7:493-499.

Desmadryl G, Hilaire C, Vigues S, Diochot S, Valmier J (1998) Developmental regulation of T-, $\mathrm{N}$ - and L-type calcium currents in mouse embryonic sensory neurones. Eur J Neurosci 10:545-552.

Djouhri L, Bleazard L, Lawson SN (1998) Association of somatic action potential shape with sensory receptive properties in guinea-pig dorsal root ganglion neurones. J Physiol (Lond) 513:857-872.

Koltzenburg M, Stucky CL, Lewin GR (1997) Receptive properties of mouse sensory neurons innervating hairy skin. J Neurophysiol 78:1841-1850.

Lee JH, Gomora JC, Cribbs LL, Perez-Reyes E (1999) Nickel block of three cloned T-type calcium channels: low concentrations selectively block alpha1H. Biophys J 77:3034-3042.

Lewin GR, Ritter AM, Mendell LM (1992) On the role of nerve growth factor in the development of myelinated nociceptors. J Neurosci 12:1896-1905.

Lindsay RM (1988) Nerve growth factors (NGF, BDNF) enhance axonal regeneration but are not required for survival of adult sensory neurons. J Neurosci 8:2394-2405.

Perez-Reyes E (1999) Three for T: molecular analysis of the low voltageactivated calcium channel family. Cell Mol Life Sci 56:660-669.

Ritter AM, Mendell LM (1992) Somal membrane properties of physiologically identified sensory neurons in the rat: effects of nerve growth factor. J Neurophysiol 68:2033-2041.

Schroeder JE, Fischbach PS, McCleskey EW (1990) T-type calcium channels: heterogeneous expression in rat sensory neurons and selective modulation by phorbol esters. J Neurosci 10:947-951.

Scroggs RS, Fox AP (1992) Calcium current variation between acutely isolated adult rat dorsal root ganglion neurons of different size. J Physiol (Lond) 445:639-658.

Shin J-B, Martinez-Salgado C, Heppenstall PA, Lewin GR (2003) A T-type calcium channel required for normal function of a mammalian mechanoreceptor. Nat Neurosci 6:724-730.

Stucky CL, Shin JB, Lewin GR (2002) Neurotrophin-4: a survival factor for adult sensory neurons. Curr Biol 12:1401-1404. 